\title{
4-Dimensional computed tomography analysis of clinical target volume displacement in adjuvant radiation of patients with gastric cancer and its implication on radiotherapy
}

\author{
JIN PENG ${ }^{1-3^{*}}$, JUN GONG $^{4 *}$, XIAOYONG WANG ${ }^{1-3 *}$, JINGJING MOU $^{1-3}, \mathrm{HUI} \mathrm{XU}^{1-3}$, \\ JING DAI $^{1-3}$, FUXIANG ZHOU ${ }^{1-3}$ and YUNFENG ZHOU ${ }^{1-3}$ \\ ${ }^{1}$ Department of Radiation and Medical Oncology, Zhongnan Hospital of Wuhan University; ${ }^{2}$ Hubei Cancer \\ Clinical Study Center; ${ }^{3}$ Key Laboratory of Tumor Biology Behavior of Hubei Province, Wuhan, Hubei 430071; \\ ${ }^{4}$ Department of Radiation and Medical Oncology, Central Hospital of Huangshi City, Huangshi, Hubei 430000, P.R. China
}

Received February 15, 2018; Accepted November 13, 2018

DOI: $\quad 10.3892 /$ ol.2019.10037

\begin{abstract}
The present study aimed to accurately measure the displacement magnitude of the radiotherapy subsite target due to respiration, and to evaluate its implication on 4-dimensional computed tomography (4D-CT) in adjuvant radiation of gastric cancer. To investigate this, 10 patients with gastric cancer receiving adjuvant radiotherapy were enrolled. 4D-CT scans were performed on all patients and respiratory signals were recorded simultaneously. The clinical target volume (CTV) and 7 regions of interest (ROIs) were delineated in all phases of the CT imaging. The displacements of all ROIs in the cephalic-caudal, anterior-posterior and left-right directions were measured and analyzed. Two sets of plans based on planning target volume 3D $\left(\mathrm{PTV}_{3 \mathrm{D}}\right)$ and $\mathrm{PTV}_{4 \mathrm{D}}$, were generated for each patient and $\mathrm{PTV}_{3 \mathrm{Dcal}}$ was calculated by expanding the non-uniform margin on $\mathrm{CTV}_{3 \mathrm{D}}$ according to the displacement analysis data. The dosimetric parameters and target volumes of the 3 radiotherapy treatment plans were compared. The displacement of the various ROIs varied widely. The mean $\mathrm{PTV}_{4 \mathrm{D}}$ was smaller than the $\mathrm{PTV}_{3 \mathrm{D}}$ and PTV $\mathrm{PDcal}_{\text {D }}$. Compared with $\mathrm{Plan}_{3 \mathrm{D}}, \mathrm{Plan}_{4 \mathrm{D}}$ reduced the mean dose of radiation to the liver and left kidney by 23.2 and $43.5 \%$, respectively. The liver volume receiving $\geq 30$ Gy and the left kidney volume receiving $\geq 20$ Gy were decreased by 10.8 and $29.7 \%$, respectively. No differences were observed in the PTV coverage and protection of organs at risk (OARs) between Plan $_{3 \mathrm{Dcal}}$ and Plan $_{4 \mathrm{D}}$. In conclusion,
\end{abstract}

Correspondence to: Dr Fuxiang Zhou, Department of Radiation and Medical Oncology, Zhongnan Hospital of Wuhan University, 169 Donghu Road, Wuchang, Wuhan, Hubei 430071, P.R. China E-mail: happyzhoufx@sina.com

${ }^{*}$ Contributed equally

Key words: stomach neoplasm, adjuvant, radiotherapy, organ at risk, treatment plan, 4-dimensional computed tomography the breathing-induced displacement patterns of the subsite targets in patients with gastric cancer vary. The individualized CTV margins of expansion based on 4D-CT lead to a decrease PTV and radiation dose to OARs. The non-uniform margins in various directions should be considered as areas for further investigation.

\section{Introduction}

Gastric cancer is the fourth most common cancer and the second leading cause of cancer-associated mortality worldwide. Every year, 1 million individuals develop gastric cancer and $>700,000$ succumb to the disease (1). It is estimated that $\sim 50 \%$ of these patients are from China, and the majority have been diagnosed at locally advanced or advanced stage $(2,3)$. Radiotherapy has been an important component of adjuvant treatment for locally advanced gastric cancer. The upper abdomen, the radiotherapy target region, is one of the regions influenced most by respiration. The displacement of organs due to respiration is the main source of uncertainty in the volume of the radiation target. A study by Kim et al (4) measured the displacement of upper abdominal organs during respiration, revealing that the displacement of the liver, kidneys and spleen ranged between 8.9 and $13.0 \mathrm{~mm}$. Another study by Hallman et al (5) quantified respiration-induced motion of clinical target volume (CTV) in patients with liver and pancreatic cancer, demonstrating that the mean distance of motion in these patients was 9.7 and $5.0 \mathrm{~mm}$, respectively. These studies indicated that the target volume varied during respiration depending on the target location. The introduction of novel radiation technologies, including intensity-modulated radiotherapy (IMRT), has led to the decrease of the radiation toxicity in normal tissue, but the problem of target motion induced by breathing remains.

Since the treatment targets in gastric cancer adjuvant radiation include several subsites with different respiratory motion amplitudes, the characteristics of the target motion of all subsites should be considered in the process of treatment planning. However, little is known about the detailed 
displacement pattern of the target volume in gastric cancer. To guarantee effective dose coverage of the target volume, a wide margin has to be added onto the CTV in order to account for the displacement induced by respiration, increasing the radiation toxicity of organs at risk (OARs).

Previously, significant toxicity associated with adjuvant chemoradiation has been a serious concern (6). To ensure safety during radiation, compromises on the coverage and dosage of the target in the final treatment plan have to be made in numerous cases. Although accurate radiotherapy has become increasingly widely implemented, there is an increasing risk of underdosing sections of the target area. Therefore, accurate radiotherapy requires more precisely delineated areas of the target and normal organ tissue, increasing the demand for accurately describing the displacement pattern of regions of interest (ROIs).

The 4-dimensional computed tomography (4D-CT) technique is one of the most reliable approaches to accurately describe the displacement caused by respiration during radiation (7). The technique has been widely used in accurate thoracic radiotherapy, particularly in lung cancer treatment (8-10). Nearly all of the associated studies reached the conclusion that a more uniform dose distribution in the target volume and a smaller internal target volume (ITV) can be obtained through the application of 4D-CT. The majority of the applications of 4D-CT in upper abdominal radiotherapy are from research on liver and pancreatic cancer (11). There are currently no reports on the application of $4 \mathrm{D}-\mathrm{CT}$ in the treatment planning of gastric cancer.

The present study was designed to examine the displacement patterns of the ROIs associated with target volume in the gastric cancer adjuvant radiation based on 4D-CT, and to investigate its value in treatment planning. Based on the analysis of ROI displacement patterns by 4D-CT, a recommendation of asymmetric expanding margins to account for target displacement in radiation for gastric cancer is proposed. The feasibility of using an easier and more reliable way to acquire accurate $\mathrm{ITV}_{3 \mathrm{D}}$ based on the displacement data from $4 \mathrm{D}-\mathrm{CT}$ is also investigated.

\section{Patients and methods}

Samples and clinical data. A total of 10 patients with gastric adenocarcinoma receiving adjuvant radiotherapy were enrolled in this study between November and December 2013. The median age was 58 (range, 49-67). The study protocol was approved by the Ethics Committee of Zhongnan Hospital (Wuhan, China; approval no. 2013018) and informed consent was obtained from all participants. The main characteristics of the patients are listed in Table I.

The inclusion criteria were as follows: i) Histologically confirmed gastric adenocarcinoma, curative gastrostomy with D2 lymph node dissection and R0 resection; ii) patients aged between 18 and 75 years at diagnosis; iii) stage T3 to T4 and/or N1 to N3 [2010 American Joint Committee on Cancer (AJCC) staging system 7th edition (11)]; iv) Eastern Cooperative Oncology Group (ECOG) performance status of $0-2$; and $v$ ) adequate bone marrow function (hemoglobin $\geq 90 \mathrm{~g} / 1$, neutrophil count $\geq 1.5 \times 10^{9} / 1$, platelet count $\geq 100 \times 10^{9} / 1$ ); adequate liver function (serum bilirubin $\leq 1.5 x$ upper limit of normal (ULN), aspartate aminotransferase (AST), and/or alanine aminotransferase (ALT) $\leq 3 \times U L N)$; and adequate renal function (serum creatinine $\leq 0.106 \mathrm{mmol} / 1$, and calculated creatinine clearance $\leq 50 \mathrm{ml} / \mathrm{min}$ ).

The primary exclusion criteria included the following: i) Receiving neoadjuvant treatment; ii) hepatic, renal, pulmonary or cardiac dysfunction; iii) severe comorbidities, such as uncontrollable diabetes mellitus, uncontrollable hypertension, myocardial infarction or unstable angina pectoris within 6 months of surgery; iv) severe postoperative complications, such as anastomotic fistula and pancreatic fistula; and v) diagnosis with another carcinoma before operation.

CT data acquisition. The patients were immobilized in a supine position with a vacuum foam pad and were advised to breathe normally. Pressure sensors (AZ-733V; Anzai Medical Co., Ltd., Tokyo, Japan) were fixed on the upper abdomen to record respiration signals. CT data were acquired using multi-slice large aperture CT (Somatom Sensation 64; Siemens Healthineers, Erlangen, Germany). The respiration cycle was spilt into 10 phases according to the signal received by the sensors. The phases included: EX0\% (beginning of exhalation), EX $25 \%$, EX50\% (middle of exhalation), EX75\%, EX100\% (end of exhalation), IN0\% (beginning of inhalation), IN25\%, IN50\% (middle of inhalation), IN75\% and IN100\% (end of inhalation). Every patient underwent a regular 3D-CT scan, followed by a $4 \mathrm{D}-\mathrm{CT}$ scan. CT data were integrated with the respiration cycle data with the MIM software (version 6.0; MIM Software Inc,. Cleveland, OH, USA). Overall, 10 series of CT images were reconstructed for each phase of respiration. All CT data were transferred to the Oncentra brachytherapy planning system (version 4.1; Elekta Instrument AB, Stockholm, Sweden). The CT image at EX50\% was treated as baseline and the $\mathrm{CT}$ images from the remaining respiration phases were fused and reconstructed.

Definition of ROIs. Based on recurrence patterns and lymphatic drainage regulations, 3D anatomy markers were drawn up for target volume delineation in the postoperative radiation of patients with gastric cancer. The radiation target volume of the regional lymph nodes (LNs) was delineated according to the consensus of adjuvant radiation for gastric adenocarcinoma in the Hubei Cancer of Zhongnan Hospital.

$C T V$. The CTV is composed of 3 areas: Tumor bed, anastomosis and draining $\mathrm{LN}$ basin. The $\mathrm{CTV}_{3 \mathrm{D}}$ was delineated upon simulation $\mathrm{CT}$. The $\mathrm{CTV}_{4 \mathrm{D}}$ was generated by combining the CTV of all 10 aforementioned breathing phases. In the present study, 7 representative ROIs were selected as the focus for displacements analysis. These were as follows: Anastomotic staples, station no. 9 LNs (LNs around the celiac artery), no. 10 LNs (splenic hilar LNs), no. 12P LNs (hepatoduodenal ligament LNs), no. 13 LNs (LNs on the posterior surface of the pancreatic head), no. 14V LNs (LNs along the superior mesenteric vein) and no. 16a2 LNs (LNs around the abdominal aorta between the upper margin of the celiac trunk and the lower margin of 
Table I. Main characteristics of the patients.

\begin{tabular}{clclcl}
\hline Patient no. & Sex & Age, years & Primary tumor location & Surgery pattern & Staging (AJCC 6th) \\
\hline 1 & Male & 60 & Proximal & D1 & pT3N2M0 \\
2 & Male & 62 & Proximal & D2 & pT4bN3M0 \\
3 & Female & 61 & Distal & D2 & pT4aN3aM0 \\
4 & Male & 49 & Distal & D2 & pT2N1M0 \\
5 & Male & 67 & Body and proximal & D1 & pT3N+M0 \\
6 & Male & 57 & Distal & D2 & DT4bN2M0 \\
7 & Male & 49 & Distal & D2 & pT4NxM0 \\
8 & Female & 58 & Proximal & D2 & pT3N2M0 \\
9 & Male & 57 & Distal and body & D2 & pT3N1M0 \\
10 & Female & 60 & Body & & PN3 \\
\hline
\end{tabular}

AJCC, American Joint Committee on Cancer; D1, Dissection of all the Group 1 nodes; D2, Dissection of all the Group 1 and Group 2 nodes (12).

the left renal vein). The LN stations were defined according to the Japanese Gastric Cancer Association (12). The ROIs in the present study were composed of the main area of the CTV for adjuvant radiation of gastric cancer. Each ROI was delineated separately.

ITV. ITV was mainly used to account for the target displacement attributed to breathing and organ movements during radiation. In this study, the $\mathrm{ITV}_{4 \mathrm{D}}$ was defined to be equal to $\mathrm{CTV}_{4 \mathrm{D}} \cdot \mathrm{ITV}_{3 \mathrm{D}}$ was generated by adding a margin of $1 \mathrm{~cm}$ radial, $1.5 \mathrm{~cm}$ distal and $1 \mathrm{~cm}$ proximal to the $\mathrm{CTV}$, according to the recommendation of the European Organization for Research and Treatment of Cancer (EORTC) (13). For ITV 3Dcal $_{\text {, a margin }}$ recommended based on the present displacement analysis was used, as described next.

Planning target volume (PTV). The PTV encompassed ITV plus a setup error. The setup error is set as $5 \mathrm{~mm}$ in Zhongnan Hospital (evaluated by cone beam CT). The PTV $\mathrm{V}_{4 \mathrm{D}}$ was calculated by applying an expansion of $5 \mathrm{~mm}$ on the $\mathrm{ITV}_{4 \mathrm{D}}$. For the $\mathrm{PTV}_{3 \mathrm{D}}$ and PTV $\mathrm{PDcal}_{\text {D }}$, the linear sum of all errors is an overestimate of the total errors in in the majority of cases. According to the 62 nd Report of the International Commission on Radiation Units and Measurements (14), the PTV $\mathrm{PD}_{3 \mathrm{D}}$ and $\mathrm{PTV}_{3 \mathrm{Dcal}}$ were calculated based on the formula: $\mathrm{PTV}=\mathrm{CTV}+\left(\mathrm{IM}^{2}+\mathrm{SM}^{2}\right)^{1 / 2}$, where IM refers to the internal margin and SM is the setup margin.

Surgery. All patients had previously undergone curative resection with D2 LN dissection (12). This procedure involved the resection of the perigastric LNs, the left gastric artery, the common hepatic artery, the celiac artery, the splenic hilum and the splenic artery LNs. Pathological evaluation was performed on $\geq 15$ LNs.

Treatment plans. All treatment plans were developed using the Oncentra treatment plan system. In total, 3 sets of treatment plans using IMRT technology were designed for each patient: Plan $_{3 \mathrm{D}}$, Plan 3 Dcal and Plan $_{4 \mathrm{D}}$. The prescription dose of radiation was 45 Gy in 25 fractions, and 7-9 coplanar and/or non-coplanar beams were used. The plans were optimized to obtain satisfactory dose coverage, with $\geq 95 \%$ of the PTV receiving the prescribed dose. The treatment plans were delivered with 6-MV photon beams.

Plan evaluation and dose limitation. A dose-volume histogram was used to analyze the coverage of the target volume, where $\mathrm{VN}$ was the volume of the ROI that received $\geq \mathrm{N}$ Gy, $\mathrm{D}_{\max }$ was the maximum dose administered to the $\mathrm{ROI}$, and $\mathrm{D}_{\text {mean }}$ was the mean dose. The main dose limitations for OARs were as follows: Liver, V30<50\%; kidney, $\mathrm{D}_{\text {mean }}<20 \mathrm{~Gy}$ or $\mathrm{V} 20<30 \%$ and $\mathrm{V} 5<65 \%$ for both; remnant gastric, $\mathrm{V} 40<50 \%$ (no hot spot on it) and $\mathrm{D}_{\max }<54 \mathrm{~Gy}$; spinal cord, V45<0.03 $\mathrm{cm}^{3}$; intestinal (delineated upon simulation CT from $2 \mathrm{~cm}$ above PVT in cephalic-caudal direction), $\mathrm{D}_{\text {mean }}<30 \mathrm{~Gy}, \mathrm{~V} 50<10 \%$ (no hot spots on it) and $\mathrm{D}_{\max }<54 \mathrm{~Gy}$. For patients with proximal gastric cancer, a dose limitation of $\mathrm{V} 30<30 \%$ for the heart and V $20<20 \%$ for the lungs was applied.

Displacement of ROIs. The displacement was measured in three directions: Left-right (LR) direction, anterior-posterior (AP) direction and cephalic-caudal (CC) direction. In order to compare the magnitudes of displacement in the different directions in 3D, a displacement vector (DV) was defined to quantify the displacement of all ROIs, calculated with the following formula: $\mathrm{DV}=\left(\Delta \mathrm{X}^{2}+\Delta \mathrm{Y}^{2}+\Delta \mathrm{Z}^{2}\right)^{1 / 2}$, where $\Delta \mathrm{X}, \Delta \mathrm{Y}$ and $\Delta \mathrm{Z}$ represent the maximum displacement distances on the $\mathrm{x}-, \mathrm{y}-$ and z-axis, respectively.

Statistical analysis. Statistical analysis was performed using SPSS version 19.0 software (IBM Corp., Armonk, NY, USA). Analysis of variance was used for comparison among groups and variables. Multiple comparisons between the groups were performed using SNK method. $\mathrm{P} \leq 0.05$ was considered to indicate a statistically significant difference. The results are presented as the mean \pm standard deviation. The $95 \%$ confidence interval (CI) of the displacement was calculated with the following formula: CI $(95 \%)=\bar{X} \pm S * z_{\alpha / 2}$, where $\bar{X}$ refers to mean, $\mathrm{S}$ refers to standard deviation, $z_{\alpha / 2}=2.262$. 
Table II. Displacement vectors of regions of interest for the 10 patients (mean \pm standard deviation).

Displacement dimension, $\mathrm{mm}$

\begin{tabular}{lcccc}
\cline { 2 - 4 } Region of interest & Left-right (x) & Anterior-posterior (y) & Cephalic-caudal (z) & Displacement vector \\
\hline No. 9 LNs & $2.2 \pm 1.2$ & $3.8 \pm 2.2$ & $4.2 \pm 2.2$ & $9.6 \pm 1.8$ \\
No. 10 LNs & $3.3 \pm 2.5$ & $4.2 \pm 2.3$ & $7.6 \pm 3.1$ & $14.1 \pm 2.4$ \\
No. 12P LNs & $3.4 \pm 2.1$ & $5.3 \pm 2.7$ & $7.8 \pm 3.6$ & $14.6 \pm 1.6$ \\
No. 13 LNs & $2.6 \pm 1.8$ & $4.9 \pm 2.1$ & $7.1 \pm 2.9$ & $12.8 \pm 1.7$ \\
No. 14V LNs & $2.9 \pm 2.4$ & $4.6 \pm 2.4$ & $5.4 \pm 3.1$ & $12.5 \pm 2.3$ \\
No. 16a2 LNs & $1.7 \pm 1.1$ & $3.1 \pm 1.5$ & $4.7 \pm 2.2$ & $9.1 \pm 1.1$ \\
Surgical staples & $5.5 \pm 2.5$ & $3.0 \pm 1.8$ & $6.7 \pm 3.6$ & $13.5 \pm 3.7$ \\
\hline
\end{tabular}

LNs, lymph nodes.

Table III. Recommended safety margins for the regions of interest.

Margin dimension, $\mathrm{mm}$

\begin{tabular}{lccc}
\cline { 2 - 4 } Region of interest & Left-right (x) & Anterior-posterior (y) & Cephalic-caudal (z) \\
\hline No. 9 LNs & 7.0 & 8.5 & 9.0 \\
No. 10 LNs & 8.5 & 9.0 & 12.5 \\
No. 12P LNs & 8.0 & 10.0 & 13.0 \\
No. 13 LNs & 7.5 & 9.0 & 11.5 \\
No. 14V LNs & 8.0 & 9.0 & 10.0 \\
No. 16 LNs & 7.0 & 8.0 & 9.0 \\
Surgical staples & 10.5 & 8.0 & 11.5 \\
\hline
\end{tabular}

LNs, lymph nodes.

\section{Results}

Displacement of ROIs. The displacement ranged widely for various ROIs. In the same direction, the anastomosis staples exhibited the largest displacement magnitude on the LR direction (X-axis; $5.5 \pm 2.5 \mathrm{~mm}$ ), while no. $12 \mathrm{p} \mathrm{LNs} \mathrm{went} \mathrm{through} \mathrm{the}$ largest displacement on the AP direction (Y-axis; $5.3 \pm 2.7 \mathrm{~mm}$ ) and the cephalic-caudal (CC) direction (Z-axis; 7.8 $\pm 3.6 \mathrm{~mm}$ ). A significant difference in the DV was observed among the 7 ROIs $(\mathrm{P}<0.001)$. The no. 12p LNs had the largest DV, while the no. 16a2 LNs had the smallest (Table II).

Recommended margin for expanding the CTV. The $95 \%$ confidence interval (CI) was obtained based on the data of the displacement of the ROIs. The PTV $\mathrm{PDcal}_{3 \text { was }}$ calculated according to the displacement analysis data by expanding the margins of the $\mathrm{CTV}_{3 \mathrm{D}}$, according to the formula $\mathrm{PTV}_{3 \mathrm{Dcal}}=\mathrm{CTV}+\left(\mathrm{IM}^{2}+\mathrm{SM}^{2}\right)^{1 / 2}, \mathrm{IM}$ was generated by taking the upper limit value, and SM was equal to $5 \mathrm{~mm}$. Thus, the recommended safety margins for ROIs were obtained and are presented in Table III.

Comparison of the PTVs. The 3 types of PTV were compared in each patient (Fig. 1A). The volume of $\mathrm{PTV}_{4 \mathrm{D}}$ had no significant difference compared with that of PTV $\mathrm{PDcal}(868.87 \pm 318.18$ vs. 967.87 $\left.\pm 384.81 \mathrm{~cm}^{3} ; \mathrm{P}=0.538\right)$. The $\mathrm{PTV}_{3 \mathrm{D}}(1719.04 \pm 509.35)$ was the largest, significantly so compared with the PTV $\mathrm{PD}_{3 \mathrm{D} a l}$ or $\mathrm{PTV}_{4 \mathrm{D}}\left(\mathrm{P}<0.001\right.$; Fig. 1B). All PTV $\mathrm{PDcal}_{\text {Dal }}$ and $\mathrm{PTV}_{4 \mathrm{D}}$ were included within the $\mathrm{PTV}_{3 \mathrm{D}}$. For the majority of patients $(8 / 10)$, the $\mathrm{PTV}_{4 \mathrm{D}}$ was completely encompassed in the PTV $\mathrm{PDCal}_{3 \mathrm{a}}$ (Fig. 2A-C), while the $\mathrm{PTV}_{3 \mathrm{Dcal}}$ in certain sections was smaller than the $\mathrm{PTV}_{4 \mathrm{D}}$ in 2 out of 10 patients (Fig. 2D-F). However, the $\mathrm{PTV}_{3 \mathrm{D}}$ was larger than the $\mathrm{PTV}_{4 \mathrm{D}}$ in all patients.

Dosage analysis in the PTVs. The 3 treatment plans $\left(\operatorname{Plan}_{3 \mathrm{D}}\right.$, Plan $_{4 \mathrm{D}}$ and Plan 3 Dcal $)$ for all patients were compared. Following the analysis of the DVH and a slice-by-slice examination, no difference in the coverage and distribution was detected in the 3 types of treatment plan for all patients.

Dose of radiation on the OARs. The radiation doses on the OARs in the 3 types of treatment plan were compared. The $\mathrm{D}_{\text {mean }}$ and V30 of the liver showed no significant difference between the $\mathrm{PTV}_{3 \mathrm{Dcal}}$ and the $\mathrm{PTV}_{4 \mathrm{D}}$ treatment plans. The $\mathrm{D}_{\text {mean }}$ and V30 of the liver were significantly increased in the PTV treatment plan compared with those in $\mathrm{PTV}_{4 \mathrm{D}}$ and $\mathrm{PTV}_{3 \mathrm{Dcal}}$ treatment plans. The $\mathrm{D}_{\text {mean }}$ of the kidney (right and left) were significantly lower in $\mathrm{PTV}_{4 \mathrm{D}}$ and $\mathrm{PTV}_{3 \mathrm{Dcal}}$ compared with these 

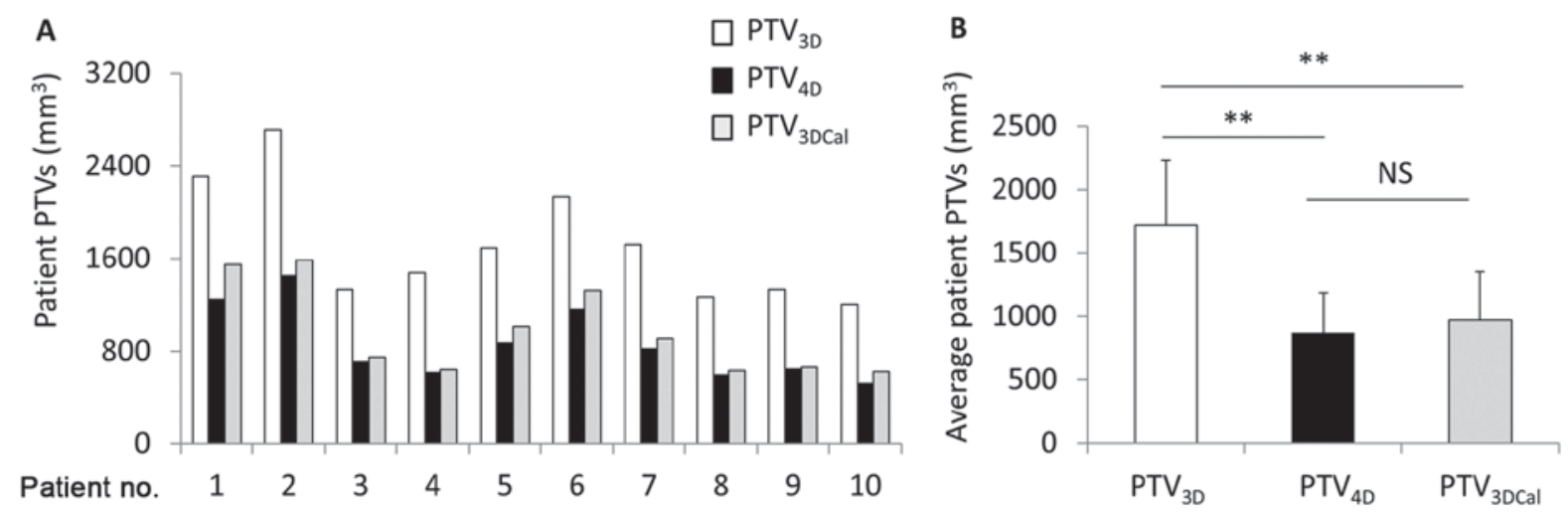

Figure 1. Comparison of the 3 types of PTV in the 10 patients. (A) The $\mathrm{PTV}_{3 \mathrm{DC} \text { Cal }}, \mathrm{PTV}_{4 \mathrm{D}}$ and $\mathrm{PTV}_{3 \mathrm{D}}$ in each patient. (B) The mean PTV $\mathrm{PDCa}_{3 \mathrm{l}}, \mathrm{PTV}_{4 \mathrm{D}}$ and PTV $3 \mathrm{D}$ in the 10 patients. $\mathrm{PTV}$, planning target volume ${ }^{* *} \mathrm{P}<0.01$.
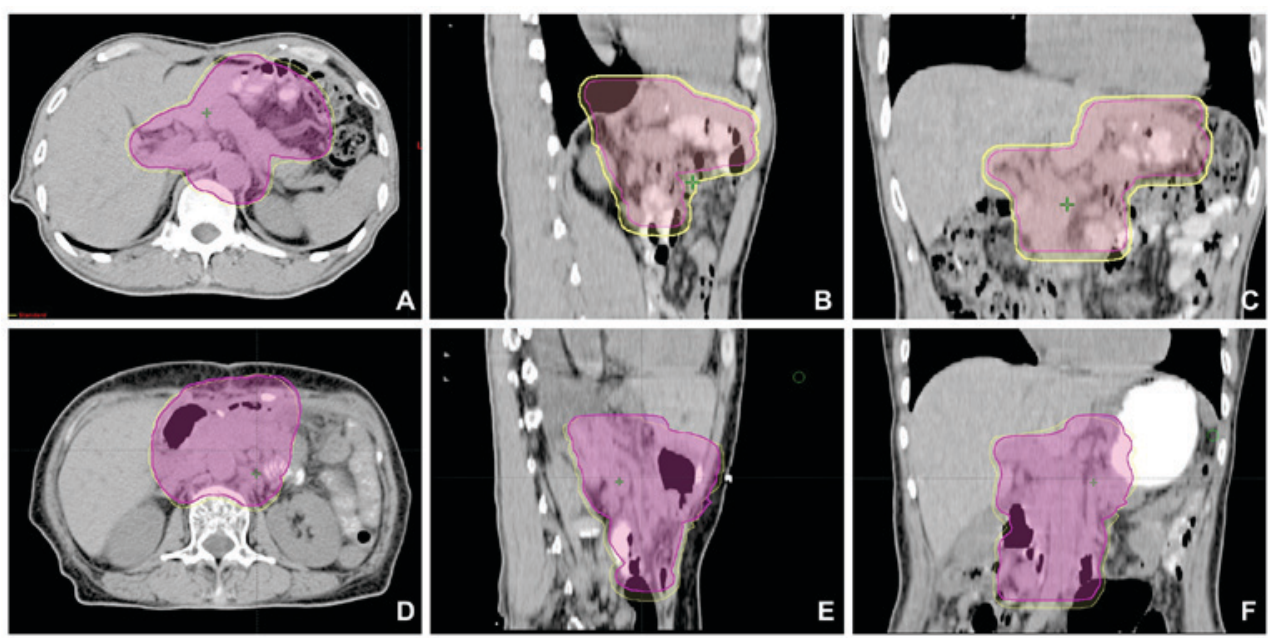

Figure 2. Representative comparison of $\mathrm{PTV}_{4 \mathrm{D}}$ and $\mathrm{PTV}_{3 \mathrm{DCal}}$ section by section. (A) Axial view, (B) coronal view and (C) sagittal view of the abdominal area in patient no. 7, revealing a larger PTV 3 Dcal compared with the PTV $\mathrm{PD}_{4 \mathrm{D}}$ in all sections. (D) Axial view, (E) coronal view and (F) sagittal view of the abdominal area in patient 9, indicating that the $\mathrm{PTV}_{3 \mathrm{Dcal}}$ is smaller than the $\mathrm{PTV}_{4 \mathrm{D}}$ in certain sections. Yellow area, PTV $3 \mathrm{DCal}$; purple area, PTV $4 \mathrm{D}$. PTV, planning target volume.

in $\mathrm{PTV}_{3 \mathrm{D}}$ treatment plan. However, the V20 of the right kidney showed no difference when $\mathrm{PTV}_{3 \mathrm{D}}$ was compared with $\mathrm{PTV}_{4 \mathrm{D}}$ or PTV 3 Dcal (Fig. 3).

\section{Discussion}

The main findings of the present study were as follows: i) Different displacement magnitudes and directions were described for each ROI in the adjuvant radiation treatment of patients with gastric cancer, implying that a uniform margin expansion for all target areas is not optimal for the generation of the PTV; ii) the $\mathrm{PTV}_{4 \mathrm{D}}$ was smaller than the $\mathrm{PTV}_{3 \mathrm{D}}$; iii) the treatment plan of $\mathrm{PTV}_{4 \mathrm{D}}$ was beneficial for the protection of the liver and left kidney; and iv) regarding the PTV and protection of OARs, Plan 3 Dcal lay between Plan $_{4 \mathrm{D}}$ and Plan ${ }_{3 \mathrm{D}}$, with a satisfactory PTV coverage, despite demonstrating certain PTV underestimation in ROIs with a large displacement range compared with that of Plan4D.

While a number of previous studies have addressed the displacement mode of upper abdominal organs or surgical staples $(4,5,15)$, the present study, to the best of our knowledge, described for the first time the displacement mode of radiotherapy-associated lymphatic drainage stations of gastric cancer. The present findings revealed that different subsites of the target volume had different patterns of displacement, according to their anatomical positions. Generally, the displacement magnitude was much larger in the CC direction. The $\mathrm{LN}$ regions adjacent to the hepatic portal and splenic hilum exhibited a larger displacement in the $\mathrm{CC}$ direction, as they are located next to organs affected by the motion of breathing. In addition to the displacement in the $\mathrm{CC}$ direction, the anastomosis staple also demonstrated a large displacement in the LR direction, which may be due to the peristalsis of the stomach. Certain ROIs, including no. 9 and no. 12 LNs, exhibited much smaller displacement than anticipated, as they are located in the peritoneum and are fixed by the surrounding ligament. These findings should be taken under consideration in the process of expanding the margins of CTV to generate the PTV, as those areas with large displacement magnitude could be administered an insufficient radiation dosage, and wide margins should be considered to account for any displacement. However, for the areas with smaller displacement, a smaller margin is sufficient to cover the target volume and this could lead to a decrease in radiation toxicity. 
A
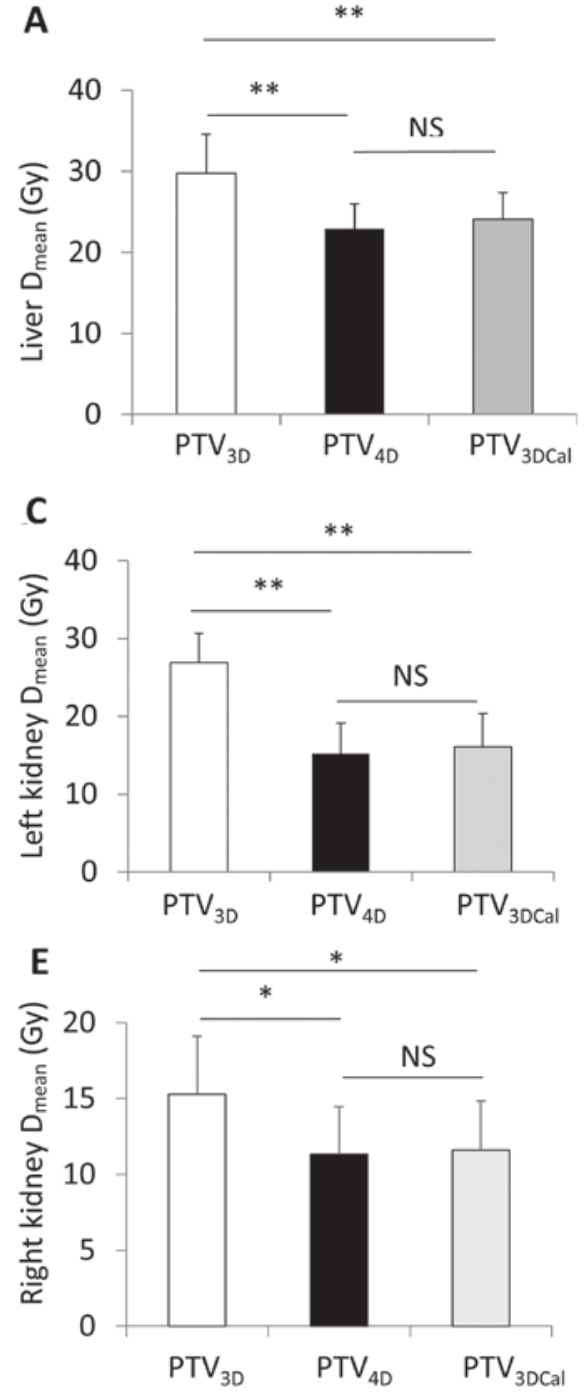

B

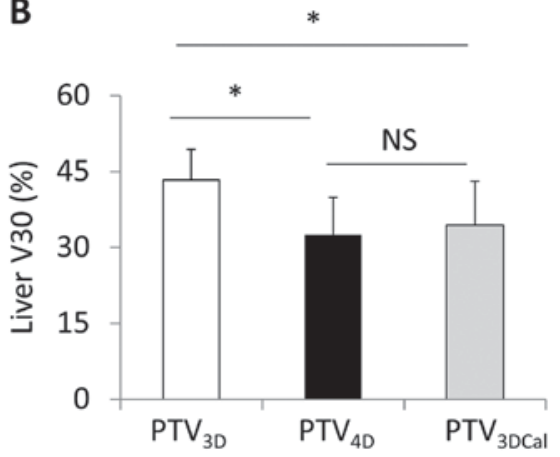

D

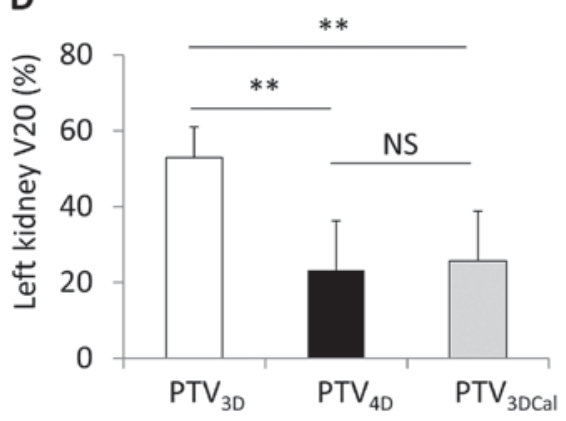

$\mathbf{F}$

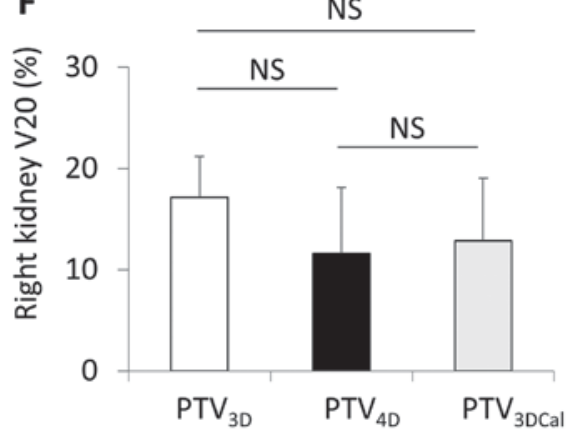

Figure 3. Comparison of 3 different doses of the liver and kidney of PTV. For PTV $\mathrm{PD}_{\mathrm{D}}$, (A) the $\mathrm{D}_{\text {mean }}$ and (B) the V30 of the liver were significantly decreased compared with that in the PTV $3 \mathrm{D}$ and $\mathrm{PTV}_{3 \mathrm{Dcal}}$. While the $\mathrm{D}_{\text {mean }}$ and V30 in liver were decreased slightly in the PTV $4 \mathrm{D}$ compared with that in the PTV $\mathrm{VD}_{3 \mathrm{Dal}}$, there was no significant difference. (C) The $\mathrm{D}_{\text {mean }}$ and (D) the V20 of the left kidney were also significantly decreased. (E) The $\mathrm{D}_{\text {mean }}$ of the right kidney was significantly decreased in $\mathrm{PTV}_{3 \mathrm{Dcal}}$ and $\mathrm{PTV}_{4 \mathrm{D}}$ compared with those in PTV3D groups. (F) The V20 of the right kidney showed no significantly difference among the three types of PTV. ${ }^{*} \mathrm{P}<0.05,{ }^{* *} \mathrm{P}<0.01$. PTV, planning target volume; $\mathrm{D}_{\text {mean }}$, mean dose; V20, volume of the organ that received $\geq 20 \mathrm{~Gy}$; V 30 , volume of the organ that received $\geq 30 \mathrm{~Gy}$.

The ITV was used to account for variations induced by the target displacement and deformation. These variations generally consist of two types, the interfractional and intrafractional variations. Interfractional variations comprise the changes in organ volume, including bowel/stomach variations, and weight, while the sources of intrafractional variations mainly come from respiration and peristalsis. Of all variations, the displacement induced by breathing is the main source of uncertainty in the adjuvant radiation for gastric cancer. As the main ROIs in the present study were the draining $\mathrm{LN}$ regions, which are less influenced by organ filling or weight changes, the interfractional variations were ignored, and the combination of $\mathrm{CTV}_{4 \mathrm{D}}$ was defined as $\mathrm{ITV}_{4 \mathrm{D}}$.

In the dosimetric analysis, no differences were observed in the PTV coverage and distribution among the three treatment plans, while the volume of $\mathrm{PTV}_{4 \mathrm{D}}$ was the smallest among the 3 sets of treatment plans. As similar studies on gastric cancer are few in number, only certain studies addressing other upper abdominal tumors could be reviewed. Matoba et al (16) compared treatment plans based on 4D- and 3D-CT in gastric lymphoma, and concluded that $\mathrm{Plan}_{4 \mathrm{D}}$ was as effective as $\operatorname{Plan}_{3 \mathrm{D}}$ and could minimize the exposure of the OARs to radiation. Xi et al (17) reached a similar conclusion regarding the radiation treatment of liver cancer. It is worth noting that, although the $\mathrm{PTV}_{4 \mathrm{D}}$ is significantly smaller than the $\mathrm{PTV}_{3 \mathrm{D}}$ and $\mathrm{PTV}_{3 \mathrm{DC} \text { Cal }}$, it is closer to the $\mathrm{PTV}_{3 \mathrm{DCal}}$ than to the $\mathrm{PTV}_{3 \mathrm{D}}$. The difference between $\mathrm{PTV}_{\mathrm{D}}$ and $\mathrm{PTV}_{3 \mathrm{D}}$ is $>800 \mathrm{~cm}^{3}$, while that between $\mathrm{PTV}_{4 \mathrm{D}}$ and $\mathrm{PTV}_{3 \mathrm{Dcal}}$ is $<100 \mathrm{~cm}^{3}$, indicating that the PTV 3 Dcal also has potential in protecting the normal organs and tissues from radiation. Furthermore, the present study confirmed that the expansion margin according to the recommendation of the EORTC was sufficient to cover the displacement induced by respiration in all patients. However, the margin may be considered too large in certain target areas, where $\mathrm{PTV}_{3 \mathrm{Dcal}}$ and $\mathrm{PTV}_{4 \mathrm{D}}$ are significantly superior to $\mathrm{PTV}_{3 \mathrm{D}}$ in protecting normal tissues.

The results of the present study demonstrate that $4 \mathrm{D}-\mathrm{CT}$ has an advantage over traditional 3D-CT with regard to obtaining 
a more accurate target volume in treatment planning. However, treatment planning based on 4D-CT has certain limitations. 4D-CT demands higher requirements for equipment and personnel training. Furthermore, the delineation of the CTV on all phases of the CT image and treatment planning is more time-consuming than that in regular treatment planning. We hope to develop a novel method for performing treatment planning, based on 3D-CT, which retains the advantage of a smaller and more accurate target volume. Based on the analysis of the subsite target displacement patterns of 4D-CT, a non-uniform expansion of the CTV can be calculated and applied to generate the $\mathrm{PTV}_{\text {3Dcal }}$. This integrates the main advantage of treatment planning based on 3D-CT and 4D-CT. In the present study, the

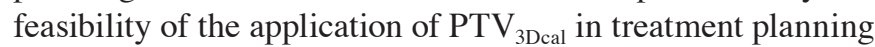
was investigated. The main result agrees with the hypothesis that the $\mathrm{PTV}_{3 \mathrm{Dcal}}$ can decrease the $\mathrm{PTV}_{3 \mathrm{D}}$ and provide better protection for OARs compared with the PTV $\mathrm{PD}_{3 \mathrm{D}}$.

However, in an analysis of target coverage of PTV $\mathrm{PDcal}_{3 \mathrm{D}}$ compared with $\mathrm{PTV}_{4 \mathrm{D}}, 2$ out of 10 patients experienced target underdosing. The upper 95\% CI limitation of target displacement was used as the recommended expansion of PTV and an estimated 5\% target underdosing was observed. The slice-by-slice dose coverage analysis revealed that the portion of $\mathrm{PTV}_{4 \mathrm{D}}$ receiving an insufficient radiation dosage was small. To reach a balance between efficacy and toxicity, a 5\% $\mathrm{PTV}_{4 \mathrm{D}}$ underdose may be considered acceptable. The investigation

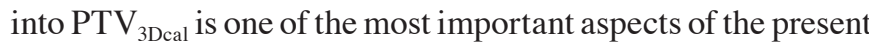
study, as it has provided a novel approach to delineation of a more accurate target volume, although certain improvements are necessary. A more accurate target displacement calculation based on a larger sample population is required, and the establishment of a more optimized mathematical model is required in order to integrate all the expansion margins.

Overall, the present study has described the detailed displacement mode of the main region of the CTV, in particular the displacement mode of the lymphatic drainage area, in the 4D-CT-based adjuvant radiation treatment of gastric cancer. A non-uniform expansion of CTV margins accounting for the displacement due to respiration is recommended, based on the displacement analysis data of ROIs. The PTV $\mathrm{PD}_{4 \mathrm{D}}$ and PTV $\mathrm{PTcal}_{3 \mathrm{D}}$ are significantly smaller than the $\mathrm{PTV}_{3 \mathrm{D}}$ and the treatment plans based on these are beneficial in protecting OARs. The $\mathrm{PTV}_{\text {3Dcal }}$ treatment planning procedure may provide a novel, simple approach to delineation of a more accurate target volume, although certain further improvements are required.

\section{Acknowledgements}

Not applicable.

\section{Funding}

This study was supported by grants from the National Natural Science Funds of China (grant nos. 81172129 and 81472798).

\section{Availability of data and materials}

The datasets used or analysed during the current study are available from the corresponding author on reasonable request.

\section{Authors' contributions}

JP and JG performed the research and wrote the manuscript. $\mathrm{JM}$ and XW collected and analysis data. HX collected and analysis the clinical data; FZ designed the project and final approved the manuscript. JD and YZ analysis part data, and reviewed the manuscript. All authors read and approved the final manuscript.

\section{Ethics approval and consent to participate}

The study protocol was approved by the Ethics Committee of Zhongnan Hospital (Wuhan, China) (approval no. 2013018) and written informed consent was obtained from all participants.

\section{Patient consent for publication}

All patients enrolled in the present study provided written consent for publication.

\section{Competing interests}

The authors declare that they have no competing interests.

\section{References}

1. Torre LA, Bray F, Siegel RL, Ferlay J, Lortet-Tieulent J and Jemal A: Global cancer statistics, 2012. CA Cancer J Clin 65: 87-108, 2015.

2. Ferlay J, Shin HR, Bray F, Forman D, Mathers C and Parkin DM: Estimates of worldwide burden of cancer in 2008: GLOBOCAN 2008. Int J Cancer 127: 2893-2917, 2010.

3. Chen W, Zheng R, Baade PD, Zhang S, Zeng H, Bray F, Jemal A, Yu XQ and He J: Cancer statistics in China, 2015. CA Cancer J Clin 66: 115-132, 2016.

4. Kim YS, Park SH, Ahn SD, Lee JE, Choi EK, Lee SW, Shin SS, Yoon SM and Kim JH: Differences in abdominal organ movement between supine and prone positions measured using four-dimensional computed tomography. Radiother Oncol 85: 424-428, 2007.

5. Hallman JL, Mori S, Sharp GC, Lu HM, Hong TS and Chen GT: A four-dimensional computed tomography analysis of multiorgan abdominal motion. Int J Radiat Oncol Biol Phys 83: 435-441, 2012.

6. Macdonald JS, Smalley SR, Benedetti J, Hundahl SA, Estes NC, Stemmermann GN, Haller DG, Ajani JA, Gunderson LL, Jessup JM and Martenson JA: Chemoradiotherapy after surgery compared with surgery alone for adenocarcinoma of the stomach or gastroesophageal junction. N Engl J Med 345: 725-730, 2001.

7. Jiang SB: Radiotherapy of mobile tumors. Semin Radiat Oncol 16: 239-248, 2006.

8. De Ruysscher D, Faivre-Finn C, Nestle U, Hurkmans CW, Le Péchoux C, Price A and Senan S: European organisation for research and treatment of cancer recommendations for planning and delivery of high-dose, high-precision radiotherapy for lung cancer. J Clin Oncol 28: 5301-5310, 2010.

9. Li FX, Li JB, Zhang YJ, Liu TH, Tian SY, Xu M, Shang DP and Ma CS: Comparison of the planning target volume based on three-dimensional CT and four-dimensional CT images of non-small-cell lung cancer. Radiother Oncol 99: 176-180, 2011.

10. Wang L, Hayes S, Paskalev K, Jin L, Buyyounouski MK, Ma CC and Feigenberg S: Dosimetric comparison of stereotactic body radiotherapy using 4D CT and multiphase CT images for treatment planning of lung cancer: Evaluation of the impact on daily dose coverage. Radiother Oncol 91: 314-324, 2009.

11. Jang JW, Brown JG, Mauch PM and Ng AK: Four-dimensional versus 3-dimensional computed tomographic planning for gastric mucosa associated lymphoid tissue lymphoma. Pract Radiat Oncol 3: 124-129, 2013.

12. Japanese Gastric Cancer Association: Japanese classification of gastric carcinoma: 3rd English edition. Gastric Cancer 14: 101-112, 2011. 
13. Matzinger O, Gerber E, Bernstein Z, Maingon P, Haustermans K, Bosset JF, Gulyban A, Poortmans P, Collette L and Kuten A: EORTC-ROG expert opinion: Radiotherapy volume and treatment guidelines for neoadjuvant radiation of adenocarcinomas of the gastroesophageal junction and the stomach. Radiother Oncol 92: $164-175,2009$

14. International Commission on Radiation Units and Measurements I: Prescribing, recording, and reporting photon beam therapy (Supplement to ICRU Report 50). ICRU Report 62, 1999.

15. Yamashita H, Okuma K, Takahashi W, Sakumi A, Haga A, Ino K, Akahane M, Ohtomo K and Nakagawa K: Four-dimensional measurement of the displacement of metal clips or postoperative surgical staples during 320-multislice computed tomography scanning of gastric cancer. Radiat Oncol 7: 137, 2012.
16. Matoba M, Oota K, Toyoda I, Kitadate M, Watanabe N and Tonami H: Usefulness of 4D-CT for radiation treatment planning of gastric MZBCL/MALT. J Radiat Res 53: 333-337, 2012.

17. Xi M, Liu MZ, Li QQ, Cai L, Zhang L and Hu YH: Analysis of abdominal organ motion using four-dimensional CT. Ai Zheng 28: 989-993, 2009 (In Chinese).

(i) (9) This work is licensed under a Creative Commons

cc) $\mathrm{EY}$ No $\mathrm{ND}$ Attribution-NonCommercial-NoDerivatives 4.0 International (CC BY-NC-ND 4.0) License. 